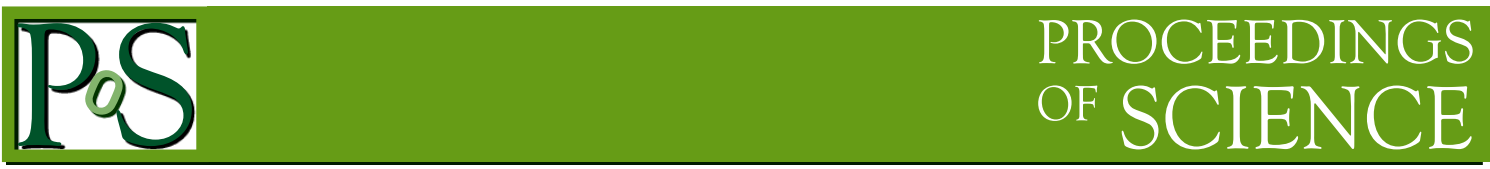

\title{
Measurements of the elastic, inelastic and total cross sections in pp collisions with ATLAS subdetectors
}

Per Grafstrom ${ }^{1}$

\section{On behalf of the ATLAS collaboration}

University of Bologna

Viale Berti Pichat 6/2,40127 Bologna, Italy

E-mail: per.grafstrom@cern.ch

Results from the ATLAS experiment at LHC on the total cross section $\left(\sigma_{\mathrm{tot}}\right)$, the inelastic cross section $\left(\sigma_{\text {inel }}\right)$, and the elastic cross section $\left(\sigma_{\mathrm{el}}\right)$ at $\sqrt{\mathrm{s}}_{\mathrm{s}}=7 \mathrm{TeV}$ are summarized . All the results are based upon measurements of the differential elastic cross section using the Roman Pot technique which allow to approach the detectors close to the beam. The results will be compared to other experiments and will also be discussed in relation to previous ATLAS measurements based upon "minimum bias" data .

The European Physical Society Conference on High Energy Physics

22-29 July 2015

Vienna, Austria

\section{${ }^{1}$ Speaker}




\section{Introduction}

This presentation summarizes results from the ATLAS [1] experiment at LHC on the total cross section $\left(\sigma_{\mathrm{tot}}\right)$, the inelastic cross section $\left(\sigma_{\mathrm{inel}}\right)$, and the elastic cross section $\left(\sigma_{\mathrm{el}}\right)$ at a centerof-mass energy of $\sqrt{s}_{s}=7 \mathrm{TeV}$. All the results are based upon measurements of the differential elastic cross section using the Roman Pot technique which allow to approach the detectors close to the beam [2]. The results will be compared to other experiments and will also be discussed in relation to previous ATLAS measurements based upon "minimum bias“ data [3].

\section{Measuring technique and the differential elastic cross section}

Elastic scattering was measured in a dedicated run at the LHC with a special high $\beta^{*}$ optics $\left(\beta^{*}=90 \mathrm{~m}\right)$ [4]. The ATLAS Roman Pots (ALFA) are installed symmetrically around the Interaction Point (IP) at a distance of $\sim 240 \mathrm{~m}$. Each side has two stations separated by $4 \mathrm{~m}$ and each station in turn has two Pots which approach the beam from below and above. All Pots are equipped with scintillating fibers.

The event selection is based upon the characteristic back-to-back topology of elastic events. As an example, figure 1a shows the correlation of the measured vertical coordinate on one side of the IP versus the other. The corresponding selection cut is also indicated in the figure. The differential elastic cross section as a function of $t$ is shown in figure $1 \mathrm{~b}$. The four momentum transfer $t$ is calculated as $-t=(p \theta)^{2}$, where $\theta$ is the scattering angle and $p$ the beam energy. The

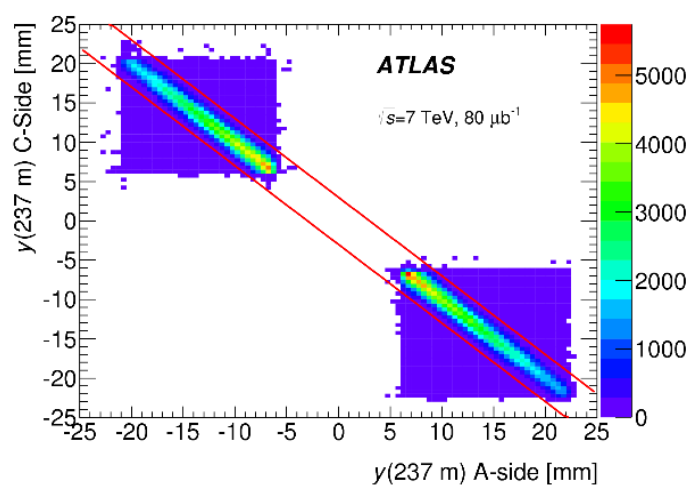

(a)

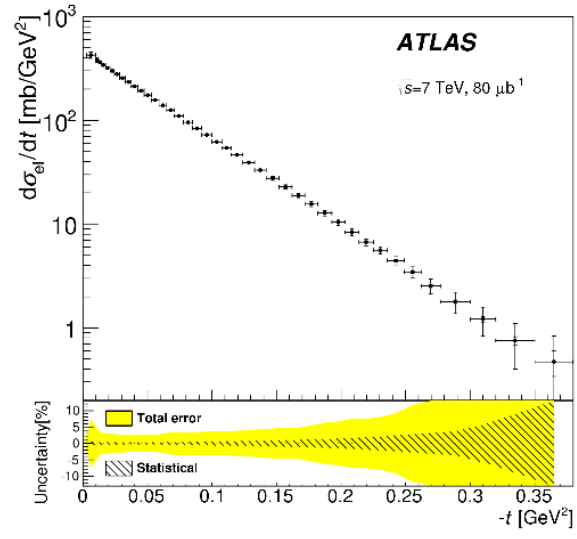

(b)

Figure 1: The correlation of the y coordinate measured at the A- and C-side for the inner stations. The stations at $237 \mathrm{~m}$ from the IP have been used. Elastic-scattering candidates after data quality, trigger and bunch selection but before acceptance and background rejection cuts are shown. Identified elastic events are required to lie between the red lines (a). The differential elastic cross section measured. The outer error bars indicate the total experimental uncertainty and the inner error bars the statistical uncertainty. The lower panel shows the relative total and statistical uncertainties (b). Figures taken from Ref. [2]. 
cross section is normalized using a measurement of the luminosity based upon the Van Der Meer technique [5]. The data has been unfolded and corrected for acceptance and background. Further corrections include reconstruction and trigger efficiencies. The main systematic uncertainty is coming from the luminosity measurement with a precision of $2.3 \%$.

\section{Results}

The total cross section is extracted from the elastic measurement using the optical theorem. The differential distribution is extrapolated to $t=0$ assuming an exponential form of the hadronic amplitude for $t$-values below $-t=0.1 \mathrm{GeV}^{2}$. A value of $\sigma_{\text {tot }}=95.4 \pm 1.4 \mathrm{mb}$ is obtained. The error includes both the statistical and systematic uncertainties of the differential measurement. In addition an extrapolation error of $0.4 \mathrm{mb}$ is included. The result of the $\sigma_{\text {tot }}$ measurement is compared in fig.2a with measurements at lower energy as well as with results using cosmic rays at higher energy. The measured total cross section is furthermore compared to the best fit to the energy evolution of the total cross section from the COMPETE Collaboration [6] assuming an energy dependence of the type $l n^{2} s$. In figure $2 b$ the result is compared to the results of the TOTEM experiment at the same energy $[7,8,9]$. TOTEM uses three different methods to extract $\sigma_{\text {tot }}$ from one unique data set. Comparing the ATLAS result with the method of TOTEM which use the same technique as ATLAS to extract $\sigma_{\text {tot }}$ gives a $1.3 \sigma$ difference between the two experiments.

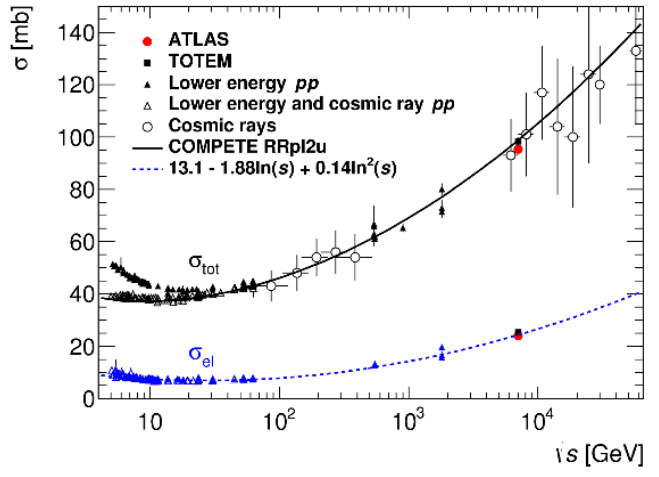

(a)

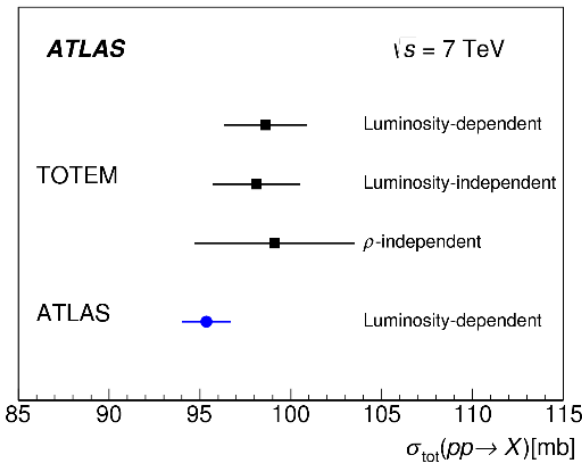

(b)

Figure 2: Comparison of total and elastic cross-section measurements presented here with other published measurements and model predictions as function of the centre-of-mass energy (a). Comparison of the presented total cross section measurement with TOTEM (b). Figures taken from Ref. [2].

The elastic cross section, $\sigma_{\mathrm{el}}$, is obtained by an integration of the differential distribution. The result is $\sigma_{\mathrm{el}}=24 \pm 0.6 \mathrm{mb}$. The value is compared to other measurements at lower energies in figure 2a. The curve seen is a second order polynomial fit in $\ln s$ of the elastic cross section. 
From a measurement of the total and elastic cross section it is possible to calculate the inelastic cross section as $\sigma_{\text {inel }}=\sigma_{\text {tot }}-\sigma_{\text {el }}=71.3 \pm 0.9 \mathrm{mb}$. In figure 3 the result is compared with all

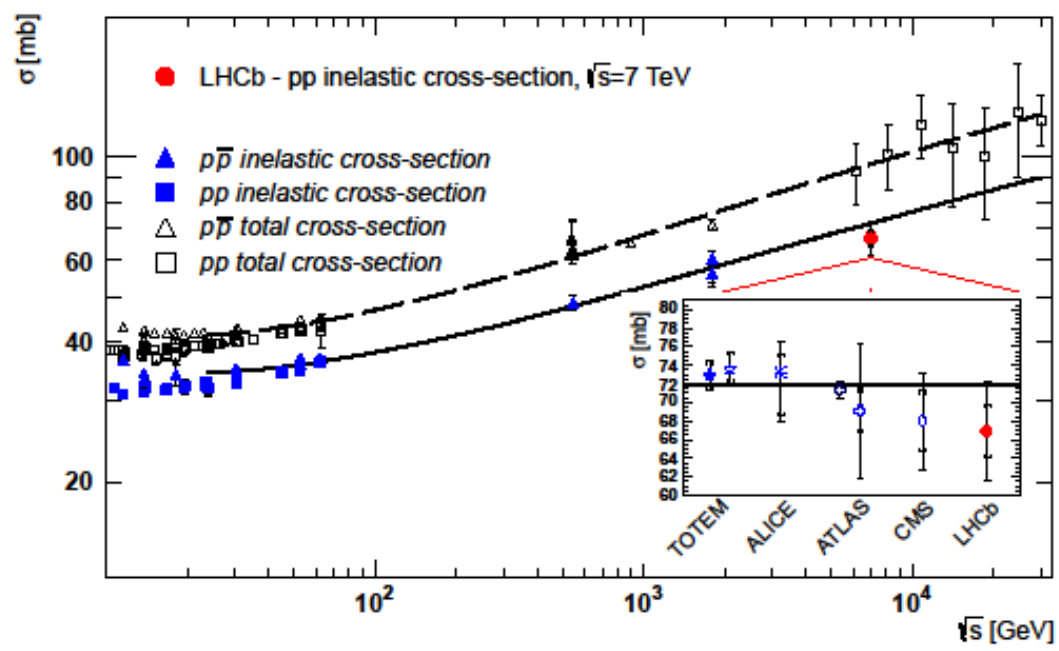

Figure 3: The main plot shows the LHCb measurement of the inelastic cross-section together with measurements at other energies. The inset is a zoom, comparing all inelastic cross-section measurements by the LHC experiments ALICE [11], ATLAS [2, 3], CMS [12] and TOTEM $[8,10]$. The ATLAS point with the smallest error bar corresponds to the measurement using the Roman Pots. The error bars give the total uncertainties of the measurements. When an inner error bar is shown, it represents the experimental uncertainties added in quadrature, while the full error bar also covers an extrapolation uncertainty. The plot is taken from [13].

other measurements of $\sigma_{\text {inel }}$ at the LHC. There is a good agreement between the different experiments and the ATLAS measurement using the Roman Pot technique clearly has the smallest error bar.

The result is also compared to a previous ATLAS measurement of $\sigma_{\text {inel }}$ using a "minimum bias” sample [3]. This measurement had a limited acceptance in the forward region which can be translated to a limitation in terms of mass of the dissociated system for diffractive events. The fiducial cross section corresponded to masses bigger than $15 \mathrm{GeV}$ and was measured to be $60.3 \pm 2.1 \mathrm{mb}$. Comparing this with the Roman Pot measurement that covers the full range of masses one gets $11.0 \pm 2.3 \mathrm{mb}$ for masses less than $15 \mathrm{GeV}$. This value is significantly larger with respect to the predictions of Monte-Carlo generators like PYTHIA and PHOJET [14,15] which ranges between 3 and $6 \mathrm{mb}$. Models like the one of Khoze, Martin and Ryskin [16] gives a better description with values in the range 11-14 mb (see discussion in ref [17]).

The slope parameter $\mathrm{B}$, characterizing the exponential decrease of the elastic distribution can also be extracted from the distribution shown in figure $1 \mathrm{~b}$ and is measured to be $\mathrm{B}=19.73 \pm 0.24 \mathrm{GeV}^{-2}$. The value is plotted in figure 4 together with the value of the slopeparameter for a number of experiments ranging from ISR to LHC. The curve drawn in the figure (from ref [18]) indicates that B increases faster with energy than the standard logarithmic energy dependence often assumed [19]. 


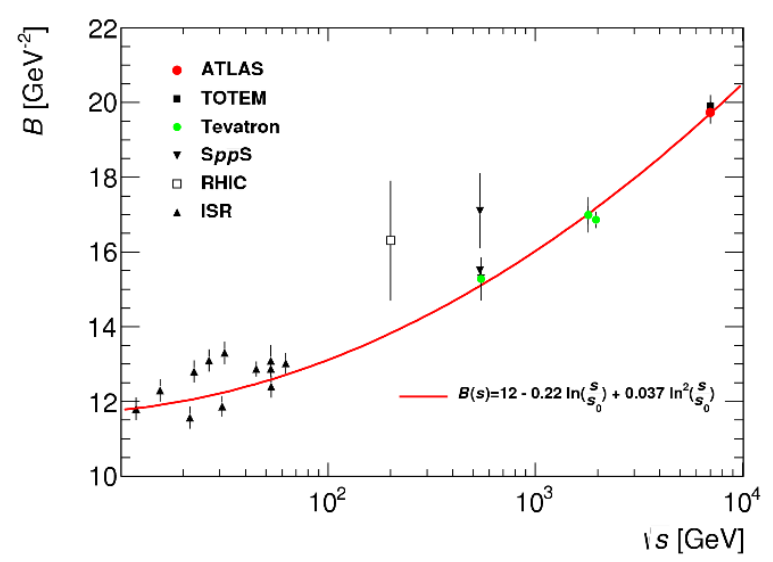

Figure 4: Comparison of the measurement of the nuclear slope B presented here with other published measurements at the ISR, at the SppS, at RHIC, at the Tevatron and with the measurement from TOTEM at the LHC. The red line shows the calculation from ref [18] which contains a linear and quadratic term in $\ln s$.

\section{Conclusions}

$\sigma_{\text {tot }}, \sigma_{\text {el }}$ and $\sigma_{\text {inel }}$ have been measured using the Roman Pot technique at a center-of-mass energy of $7 \mathrm{TeV}$ at the LHC. Results are in agreement with the TOTEM results but with slightly better precision. Concerning $\sigma_{\text {inel }}$ the result are in agreement with all other LHC experiments but again with better precision. Comparing $\sigma_{\text {inel }}$ with a previous "minimum biased" based measurement from ATLAS yield a cross section of 11.3 $\pm 2.3 \mathrm{mb}$ for masses of the dissociated system below $15 \mathrm{GeV}$. The slope parameter B increases faster with the center-of-mass energy than the "standard" In s behavior normally assumed.

\section{References}

[1] ATLAS Collaboration, JINST 3 (2008) S08003.

[2] ATLAS collaboration, Nucl.Phys. B889 (2014) 486-548.

[3] ATLAS Collaboration, Nature Commun. 2 (2011) 463.

[4] H. Burkhardt et al., Conf. Proc. C1205201 (2012) 130.

[5] S. van der Meer, ISR-PO-68-31 (1968).

[6] J. Cudell et al., (COMPETE Collaboration), Phys. Rev. Lett. 89 (2002) 201801.

[7] G. Antchev et al., (TOTEM Collaboration), Europhys. Lett. 101 (2013) 21002.

[8] G. Antchev et al., (TOTEM Collaboration), Europhys. Lett. 101 (2013) 21004.

[9] G. Antchev et al., (TOTEM Collaboration), Europhys. Lett. 101 (2013) 21003. 
[10] G. Antchev et al., (TOTEM Collaboration), Europhys. Lett. 96 (2011) 21002

[11] B. Abelev et al., (ALICE collaboration), Eur. Phys. J. C 73 (2013) 2456.

[12] CMS collaboration, Phys.Lett. B722 (2013) 5.

[13] R.Aaij et al., (LHCB collaboration), JHEP 02 (2015) 129.

[14] R. Engel, Z. Phys. C 66 (1995) 203.

[15] F. W. Bopp, R. Engel, J. Ranft, Conf. Proc. LISHEP98 (1998) 729.

[16] M. G. Ryskin, A. D. Martin, V. A. Khoze, Eur. Phys. J. C 71 (2011) 1617.

[17] ATLAS Collaboration, Eur. Phys. J. C 72 (2012) 1926.

[18] V. A. Schegelsky, M. G. Ryskin, Phys. Rev. D85 (2012) 094024

[19] A. Donnachie, P.V. Landshoff, Nucl. Phys. B231 (1984) 189. 